\title{
Syndrome $\mathrm{X}$ and hyperventilation
}

\author{
N P Lewis, S J Hutchison, N Willis, A H Henderson
}

\begin{abstract}
The cardiorespiratory responses to exercise and forced hyperventilation were measured in 17 unselected patients with syndrome $X$ (angina, positive exercise test, normal coronary arteriogram, no other cardiovascular disease) and compared with those in 15 healthy subjects. Forced hyperventilation produced hypocapnia and metabolic alkalosis but no chest pain or electrocardiographic change. Patients with syndrome $X$ showed reduced maximum oxygen consumption with an increased respiratory exchange ratio at peak exercise, confirming that exercise was limited by skeletal muscle perfusion-and thus that the increase in cardiac output with exercise is limited in syndrome $X$ as in heart failure. Arterial carbon dioxide tension $\left(\mathrm{PCO}_{2}\right)$ homoeostasis during exercise was normal but the ventilatory cost of carbon dioxide excretion was increased in syndrome $X$ (as in heart failure). End tidal $\mathrm{PCO}_{2}$ measurements correlated only poorly with arterial $\mathrm{PCO}_{2}$ in individual patients with syndrome $X$, providing a possible explanation for previous reports, based on end tidal $\mathrm{PCO}_{2}$ of inappropriate hyperventilation.

Patients with syndrome $X$ did not show inappropriate hyperventilation but they did show hyperventilation that was appropriate to maintain normal arterial $\mathrm{PCO}_{2}$ in the face of reduced cardiac reserve.
\end{abstract}

Most patients with chest pain and normal coronary arteriography have non-cardiac pain that is musculoskeletal or oesophageal in origin. $^{12} \mathrm{~A}$ few are thought to have true angina attributable by inference to an impaired coronary dilator response at the microvascular level (syndrome $\mathrm{X})^{34}$ though in the absence of a directly documented vascular abnormality or known pathogenetic mechanism the existence of this syndrome is not universally accepted. Because of this uncertainty, some have ascribed the symptoms to hyperventilation, ${ }^{5-7}$ which would accord with the low end tidal partial pressure of expired carbon dioxide. ${ }^{7}$ Hyperventilation has indeed been shown to induce electrocardiographic ST segment depression, ${ }^{8}$ and alkalosis that is associated with hyperventilation can induce coronary vasoconstriction. ${ }^{9}$ To investigate the possible contribution of hyperventilation in these patients, we studied the cardiorespiratory response to exercise and hyperventilation in an unselected group of patients with syndrome $\mathrm{X}$ (defined as typical stable effort angina, a positive exercise test, and a normal coronary arteriogram in the absence of other cardiovascular disease)

\section{Patients and methods \\ PATIENTS}

We studied 17 patients (mean age 50 years, range 40-67 years; eight men), all of whom gave an independently confirmed history considered to be typical of effort angina (mean duration 3.9 years) with no other evidence of cardiovascular disease on clinical examination, chest radiograph, echocardiogram, or left ventricular angiogram; and in all of whom the coronary arteriograms were independently confirmed as entirely normal. No patient had hypertension or diabetes. Two patients smoked, and five were ex-smokers. Eleven patients were taking regular medication (four with $\beta$ blockers, seven with calcium antagonists, four with long acting nitrates). The resting electrocardiogram was normal except in three patients with left bundle branch block. Exercise tolerance was limited in all patients, to the equivalent of New York Heart Association category II (15 patients) or III (two patients): in all patients treadmill exercise showed diagnostic ST segment depression $(\geqslant 1 \mathrm{~mm}$ horizontal or downsloping ST segment) or left bundle branch block (in five patients, exercise-related in two). All patients had normal respiratory function tests (spirometry, lung volumes, and transfer factor).

We studied 15 healthy individuals (mean age 47 years, range $22-75$ years, eight men) as controls.

\section{PROTOCOL}

Patients and controls performed maximum treadmill exercise tests (Weber or standard Bruce protocol, selected as appropriate for exercise capacity) and 3 minutes of forced hyperventilation after familiarisation with the procedures (with 30 minute rest periods preceding each procedure) $>2$ hours after a meal and $>24$ hours after stopping medication. The study was approved by the local ethics committee.

Respiratory gas exchange was measured as decribed elsewhere. ${ }^{10}$ During the first exercise test, expired gas from a face mask (Hans Rudolph 7900) was carried to a 7 litre mixing 
Table Heart rate, systolic blood pressure, maximum oxygen consumption, respiratory exchange ratio $(n=17)$ and arterial and end tidal gas measurements $(n=13)$ from both exercise tests in patients with syndrome $X$. (The mean data for patients without left bundle branch block are given in italic and in parentheses, $n=12$ and 9 respectively.)

\begin{tabular}{|c|c|c|c|}
\hline & Rest & $50 \%$ of maximum exercise & Maximum exercise \\
\hline $\begin{array}{l}\text { Heart rate (per min }) \\
\mathrm{SBP}(\mathrm{mm} \mathrm{Hg}) \\
\mathrm{Vo}_{2} \max (\mathrm{ml} / \mathrm{min} / \mathrm{kg}) \\
\mathrm{RER} \\
\mathrm{CO}_{2} \mathrm{ET}(\mathrm{mm} \mathrm{Hg}) \\
\mathrm{PaCO}_{2}(\mathrm{~mm} \mathrm{Hg}) \\
\mathrm{pH} \\
\mathrm{Bicarbonate}(\mathrm{mmol} / \mathrm{l}) \\
\mathrm{PaO}_{2}(\mathrm{~mm} \mathrm{Hg})\end{array}$ & $\begin{array}{l}81(16)(74) \\
125(13)(126) \\
-0.7(0 \cdot 1)(0.7) \\
35(4)(35) \\
37(4)(37) \\
7 \cdot 43(0.04)(7.44) \\
26(1)(26) \\
92(13)(93)\end{array}$ & $\begin{array}{l}\overline{ } \\
\bar{z} \\
\overline{38(4)(38)} \\
39(4)(39) \\
7.42(0.03)(7.41) \\
25(1)(25) \\
92(7)(91)\end{array}$ & $\begin{array}{l}135(26)(128) \\
160(22)(160) \\
20 \cdot 1(4 \cdot 4)(20 \cdot 1) \\
1 \cdot(0 \cdot 1)(1 \cdot 1) \\
37(4)(37)^{\star} \\
36(3)(36)^{\star} \\
7 \cdot 40(0 \cdot 06)(7 \cdot 39) \\
24(3)^{\star}(23) \\
100(9)^{\star}(100)\end{array}$ \\
\hline
\end{tabular}

$\mathrm{SBP}$, systolic blood pressure; $\mathrm{VO}_{2}$ max, maximum oxygen consumption; RER, respiratory exchange ratio $\left(\mathrm{VcO}_{2} / \mathrm{Vo}_{2}\right) ; \mathrm{Co}_{2} \mathrm{ET}$, end tidal $\mathrm{PCO}_{2} ; \mathrm{PaCO}_{2}$, arterial $\mathrm{PCO}_{2} ; \mathrm{PaO}_{2}$, arterial $\mathrm{Po}_{2}$.

${ }^{\star} \mathrm{p}<0.05$ compared with resting value (analysis of variance)

box (Airspec) to allow measurement of minute ventilation, oxygen consumption, and carbon dioxide elimination by argon dilution and mass spectrometry (Airspec 2200) corrected to standard temperatures and pressures dry. ${ }^{11}$ During the second exercise test and the forced hyperventilation test a capillary tube continuously sampled expired air from within the face mask to measure respiratory rate and end tidal oxygen and carbon dioxide, averaged over the sampling period of 30 seconds; end tidal carbon dioxide values were corrected as previously described. ${ }^{12}$ Heparinised arterial blood samples were taken during all these procedures (via an indwelling arterial cannula inserted 30 minutes before any measurement) for blood gas analysis in 13 of the 17 patients.

The relation of minute ventilation to minute carbon dioxide production during exercise was analysed by linear regression. Data are presented as mean (1 SD). Repeated measures in the same individual were analysed by analysis of variance. Other data were compared by paired or unpaired Student's $t$ test as appropriate. Probability values of $<0.05$ were regarded as significant.

\section{Results}

During voluntary forced hyperventilation the respiratory rate increased (from 12(5) to 31(7) per minute), arterial $\mathrm{PCO}_{2}$ and end tidal $\mathrm{PCO}_{2}$ fell (from $36(4)$ to $23(5) \mathrm{mm} \mathrm{Hg}$ and from $33(3)$ to $20(6) \mathrm{mm} \mathrm{Hg}$, respectively), and $\mathrm{pH}$ increased (from $7 \cdot 4(0 \cdot 04)$ to $7 \cdot 6(0 \cdot 10)$ ), without chest pain or electrocardiographic change in any individual.

The table shows the exercise test data for patients with syndrome $X$. The patients had similarly limited exercise duration in both tests $(10.2(2.6)$ and $9.9(2.5)$ minutes), and maximal oxygen consumption was less than in the controls $(20 \cdot 1(4 \cdot 4) v 29 \cdot 7(7 \cdot 2) \mathrm{ml} / \mathrm{min} / \mathrm{kg}$, $\mathrm{p}<0.001)$. Exercise was limited by chest pain in 14 (including two of the patients with fixed left bundle branch block and one with left bundle branch block induced by exercise), and by dyspnoea (a patient with fixed left bundle branch block), fatigue, and hypotension $(40 \mathrm{~mm} \mathrm{Hg}$ fall in systolic blood pressure in a patient with left bundle branch block induced by exercise) in one patient each. In all these patients there was diagnostic electrocar- diographic evidence of myocardial ischaemia or left bundle branch block. At maximum exercise the respiratory exchange ratio (minute carbon dioxide/minute oxygen consumption) was increased to $1 \cdot 1(0 \cdot 1)$ as in the healthy controls (compared with $0.7(0.1)$ at rest). At peak exercise, there were very small reductions in arterial $\mathrm{PCO}_{2}$, bicarbonate, and $\mathrm{pH}$, reflecting a compensated metabolic acidosis; but arterial $\mathrm{PCO}_{2}$ was normal at $50 \%$ maximum exercise. There was thus no evidence of inappropriate hyperventilation.

The relation between minute ventilation and minute carbon dioxide $\left(\mathrm{VE} / \mathrm{VCO} \mathrm{CO}_{2}\right)$ on exercise was linear in all syndrome $\mathrm{X}$ patients (all $r \geqslant 0.92$ ) as it was in the healthy controls (figure); the slope (m) of this relation was, however, significantly steeper $(32.3(4 \cdot 6) v$ $26 \cdot 2(5 \cdot 0), p<0.01)$, though the minute ventilation axis intercepts (c) were similar $(2.6(1.9) 1 / \mathrm{min} v 2 \cdot 6(2 \cdot 2) 1 / \mathrm{min})$. The ventilation required for carbon dioxide elimination was thus increased in patients-for example at a minute carbon dioxide production of $11 /$ min, it was $34 \cdot 9(4 \cdot 2)$ compared with $27 \cdot 9(4 \cdot 3)$ $1 /$ min in the healthy controls $(p<0.001)$. In individual syndrome $\mathrm{X}$ patients end tidal $\mathrm{PCO}_{2}$ correlated poorly with arterial $\mathrm{PCO}_{2}$ both at

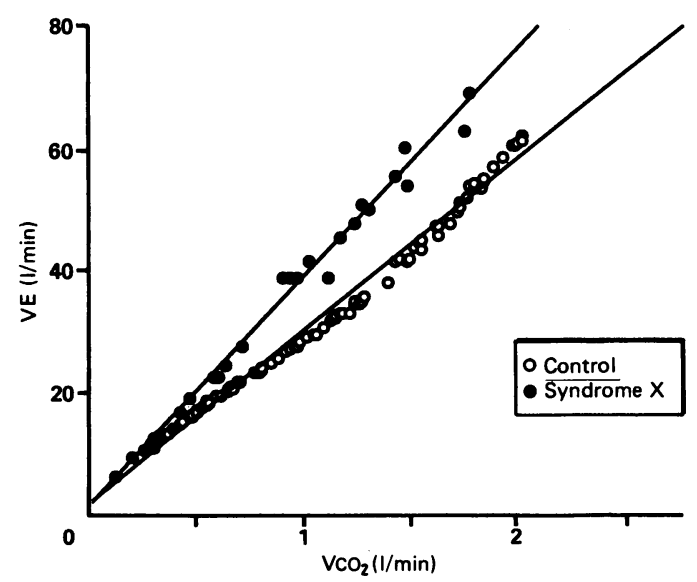

Figure Relation between minute ventilation and minute carbon dioxide production during exercise in a healthy control and a patient with syndrome $X$. Minute ventilation axis intercepts were similar in both, but the slope (37.5) axis intercepts were similar in both, but the slope (37.5)
was steeper in syndrome $X$ than in the healthy control $(28.2)$. The $r$ values of both linear regressions are 0.99 . $V E$, minute ventilation; $\mathrm{COO}_{2}$, minute carbon dioxide production. 
rest $(r=0.36, N S)$ and during exercise $(r=0 \cdot 44, \mathrm{NS})$, though it correlated well during forced hyperventilation $(r=0.86$, $\mathrm{p}<0.001$ ).

The findings did not differ in patients with left bundle branch block and without. For the 12 patients without left bundle branch block (mean age 49 years, range 40-67 years, five men) the exercise duration in the two tests were $10 \cdot 1(1 \cdot 4)$ and $9 \cdot 9(1 \cdot 8)$ minutes and exercise was limited by chest pain in 11 and fatigue in one. Haemodynamic changes, maximum oxygen consumption, and arterial gas changes during exercise were similar in the 12 patients without left bundle branch block (see table), as were the minute ventilation/minute carbon dioxide relations (slope, $\mathrm{m}=$ $33.2(4.5), \mathrm{p}<0.01$ compared with controls; ventilation axis intercept, $c=2 \cdot 7(1 \cdot 9) 1 / \mathrm{min})$.

\section{Discussion}

Since the advent of coronary angiography, reports on patients with "chest pain with normal coronary arteries" have accumulated, but the patients have not always been rigorously characterised nor have there been agreed criteria to define such patient groups. Among possible causal mechanisms for the symptoms, hyperventilation has been suggested with supportive evidence from measurements of end tidal $\mathrm{PCO}_{2}$.

We therefore investigated a group of patients selected solely because they fulfilled predefined criteria for what has come to be known as syndrome $\mathrm{X}$. We included patients with exercise induced or fixed left bundle branch block because in patients with syndrome $X$ left bundle branch block can develop; analysis of the data showed similar findings whether or not patients with left bundle branch block were included.

Voluntary forced hyperventilation caused the expected reduction in arterial $\mathrm{PCO}_{2}$ and respiratory alkalosis but it did not induce chest pain or electrocardiographic change. The patients were therefore studied during formal exercise testing. As in the healthy controls, arterial $\mathrm{PCO}_{2}$ was maintained within a narrow range by matching the ventilatory clearance of carbon dioxide to its increased metabolic rate of production. ${ }^{1314}$ At maximum exercise there was only a small reduction in arterial $\mathrm{PCO}_{2}$, compensating for the onset of anaerobic metabolism. ${ }^{1314}$ There was no evidence of altered arterial $\mathrm{PCO}_{2}$ setpoint or inappropriate hyperventilation either at rest or during exercise. Inappropriate hyperventilation thus does not seem to be the cause of symptoms in patients with syndrome $\mathrm{X}$ as here defined.

Patients with syndrome $\mathrm{X}$, however, showed reduced maximum oxygen consumption, with metabolic acidosis at peak exercise, implying that exercise was limited by perfusion to the exercising skeletal muscle as in heart failure; this is consistent with reported evidence of cardiac dysfunction in syndrome $X .^{1516}$ Moreover, the ventilatory cost of carbon dioxide clearance was increased in patients with syndrome $\mathrm{X}$, a finding also characteristic of patients with congestive heart failure. ${ }^{1718}$ The increase in carbon dioxide production resulting from the increased work of exercise, the increased respiratory exchange ratio as anaerobic metabolism developed at peak exercise, and the increased ventilation needed to excrete carbon dioxide all contributed to increased ventilation in these patients - or what might be called appropriate hyperventilation because it maintained normal arterial $\mathrm{PCO}_{2}$.

End tidal $\mathrm{PCO}_{2}$ values correlated well with arterial $\mathrm{PCO}_{2}$ values in the group as a whole, as was reported in healthy controls, but the correlation in individuals was poor (except during forced hyperventilation). This is consistent with the increase in physiological deadspace implied by the increased ventilatory cost of carbon dioxide excretion in these patients. ${ }^{13}$ Practically, it indicates that measurements of end tidal $\mathrm{PCO}_{2}$ cannot be used as an indicator of arterial $\mathrm{PCO}_{2}$ in individual patients with syndrome $\mathrm{X}$, as was done in earlier studies that of suggested that hyperventilation was a common iv cause of symptoms in patients with "chest pain" and normal coronary arteries. ${ }^{7}$

We found no evidence of inappropriate hyperventilation in patients with syndrome $X$, but we did find evidence of appropriate hyperventilation that seemed to result from an increase in physiological deadspace as is typical of other patients with reduced cardiac reserve.

1 Dart AM, Alban Davies H, Dalal J, Ruttley M, Henderson AH. "Angina" and normal coronary arteriograms: follow-up study. Eur Heart J 1980;1:97-100.

2 Proudfit WL, Shirley EK, Jones FM. Selective cine coronary arteriography: correlation with clinical findings in 1000 cases. Circulation 1986;33:901-10

3 Opherk D, Zebe H, Weihe E, et al. Reduced coronary dilatory capacity and ultrastructural changes of the myocardium in patients with angina pectoris but normal myocardium in patients with angina pectoris but

4 Cannon RO, Epstein SE. Microvascular angina as a cause of chest pain with angiographically normal coronary arteries. Am J Cardiol 1988;i:155-7.

5 Evans DW, Lum LC. Hyperventilation: an important cause of pseudoangina. Lancet 1977;i:155-7.

6 Freeman LJ, Nixon PGF. Chest pain and the hyperventilation syndrome: some aetiological considerations. Postgrad Med J 1985;61:957-61.

7 Chambers JC, Kift PJ, Gardner WN, Jackson G, Bass C Value of measuring end-tidal partial pressure of carbon dioxide as an adjunct to treadmill exercise testing. $\mathrm{Br} \mathrm{Med}$ $J 1988 ; 296: 1281-5$.

8 Lary D, Goldschlager N. Electrocardiographic changes during hyperventilation resembling myocardial ischaemi in patients with normal coronary arteriograms $A m$ Heart in patients with nor

9 Takaoka $\mathrm{K}$, Yasue $\mathrm{H}$, Horio $\mathrm{Y}$. Possible role of coronary spasm in acute myocardial infarction precipitated by hyperventilation. Br Heart $J$ 1988;59:256-8.

10 Macdougall IC, Lewis NP, Saunders MJ, et al. Long-term cardiorespiratory effects of amelioration of renal anemi by erythropoietin. Lancet 1990;335:489-93.

1 Davies NJH, Denison DM. The measurement of metabolic gas exchange and minute volume by mass spectrometry alone. Respir Physiol 1979;36:261-7.

12 Jones NL, Robertson DG, Kane JW. Differences between end-tidal and arterial $\mathrm{PCO}_{2}$ in exercise. J Appl Physiol 1979;47:954-60.

13 Jones NL. Clinical exercise testing. 3rd ed. Philadelphia: W B Saunders, 1988:13-74.

14 Whipp BJ, Ward SA. The normal respiratory response in exercise. In: Leff AR, ed. Cardiopulmonary exercise testing.

15 Wieshammer S, Delagardelle C, Sigel HA, et al. Haemodynamic response to exercise in patients with chest pain and normal coronary arteriograms. Eur Heart $J$ 1986; and normal

16 Kubler W, Opherk D. Angina pectoris with normal coronary arteries. Acta Med Scand 1985;694(suppl):55-7.

17 Fink LI, Wilson JL, Ferraro N. Exercise ventilation and pulmonary artery wedge pressure in chronic stable congestive heart failure. Am J Cardiol 1986;57:249-53.

18 Lewis NP, Fox KAA, Henderson AH. In congestive heart failure (CHF) exercise air spectroscopy provides a sensitive marker of functional status [Abstract]. Eur Heart $J$ 1989;10:438. 